\title{
Digitized optofluidic element and its application to tristate lens for presbyopia correction
}

\author{
Valdemar Portney ${ }^{\mathrm{a}},{ }^{\mathrm{a} *}$ F. Richard Christ, ${ }^{\mathrm{b}}$ and Marie Dvorak Christ ${ }^{\mathrm{b}}$ \\ ${ }^{a}$ Vision Advancement LLC, Newport Coast, California, United States \\ bnVision Biomedical Group, Inc., Irvine, California, United States
}

\begin{abstract}
Current optofluidic devices are analog focus-tunable elements with a continually varying focus between foci. An innovative digitized optofluidic element was developed to enable fast switching between the foci. The operation involves an internally placed elastic membrane of changing shape between the refractive and diffractive forms with a tiny amount of fluid transfer by a micro-actuator. This allows for switching the digitized optofluidic element between the refractive and diffractive states of the preset optical powers. A multimodal relief surface design was used in the diffractive state to manage the diffractive grooves and improve the chromatic characteristics. We applied finite element analysis to establish the membrane mechanical properties. The proof-of-concept prototypes were made using material bonding fabrication processes and were used to demonstrate an elastic membrane shape change between the refractive and diffractive forms for switching between the corresponding optical states. The design of the digitized optofluidic element allowed for bistate or tristate switching by a single actuator. The switchable element can be fairly thin, enabling conversion of a fixed lens into a dynamic lens switchable between two or three foci. The application of the tristate design to eyewear for presbyopia correction is discussed. (c) The Authors. Published by SPIE under a Creative Commons Attribution 4.0 Unported License. Distribution or reproduction of this work in whole or in part requires full attribution of the original publication, including its DOI. [DOI: 10.1117/1.OE.60.6.065103]
\end{abstract}

Keywords: focus tuning; optofluidic; lens design; eyewear.

Paper 20210231 received Mar. 4, 2021; accepted for publication Jun. 2, 2021; published online Jun. 17, 2021.

\section{Introduction}

Presbyopia is the inability of the eye to focus at far and near distances, a condition impacting a vast majority of people beginning in their forties. ${ }^{1}$ Common choices for presbyopia correction via eyewear rely on fixed lenses-a single vision lens for near correction, such as bifocal or trifocal lenses, and progressive addition lenses (PALs). Common among them is "frame independence," which allows the wearer to choose their frames as a fashion statement. The majority of presbyopes are prescribed PALs, which are most optically complex, followed by bifocal or trifocal spectacles. ${ }^{2}$ The PALs have a vertical gradient of increasing power toward the lower part such that the wearer can see at all distances by looking through different parts of the lens. Although PALs are widely used, they do not work for everyone. As the lens optical complexity increases, the optical quality limitations also increase; PALs can be very sensitive to spectacle frame mounting errors and suffer from astigmatism and optical distortion at the lens periphery; they cannot be prescribed for eyes with considerable refractive error difference or astigmatism, and they restrict the field-of-vision. ${ }^{3}$ Although the limitation is generally tolerable with lower ranges of optical power of the PAL optic, it increases with higher ranges of optical powers that are needed for older individuals. As with other senses, such as the vestibular and somatosensory systems, these degrade with age; older individuals rely more heavily on vision and have an increased risk of injuries from fall due to visual restrictions of the bifocal, trifocal and PALs., ${ }^{4,5}$

There has been an ongoing effort to develop "focus-tuning lenses" to maintain a single focus lens quality at difference distances. Among early implementations of focus-tuning lenses is the Alvarez lens, which involved a complementary pair of cubic phase plates that shifted relative to each other to allow for varying optical power. ${ }^{6}$ Other efforts include optofluidic lenses with

*Address all correspondence to Valdemar Portney, vidadv@ cox.net 
"fluid-membrane-fluid" structures to reduce the gravity effect ${ }^{7,8}$ and electrowetting lenses based on two immiscible fluids actuated by electrostatic forces. ${ }^{9}$ An approach to optofluidic spectacles offered by the Laclaree team is a fluid-filled focus-tuning lens assisted by a low-power, highvolume microfluidic actuator. ${ }^{10}$ The problematic issues with all such focus-tuning lenses are (1) the frame dependence for actuation components placement at the lens frame as a fairly significant fluid volume has to be transported and (2) the slow response time for focus adjustment between viewing distances.

Exploration of electro-active materials (liquid crystal, for instance) application for eyewear to achieve faster response times has been quite extensive. ${ }^{11-13}$ The corresponding operational principle of switching the refractive index value with an applied electric field is called material-based switchable (MBS) technology. Based on this principle, several concepts of a bistate switchable lens have been designed: parabolic gradients, solid profiles immersed inside the liquid crystal, and switching between refractive and diffractive optical states. All of these approaches share major drawbacks when considering the lens for presbyopia correction-the use of unpolarized light requires doubling the stack of active layers [one for each E-field direction] and the overall complexities resulting in frame dependency and the high cost of an MBS lens (TouchFocus ${ }^{\mathrm{TM}}$ spectacles by Mitsui Chemicals, Japan).

This paper introduces a digitized optofluidic element that combines the simplicity of the analog focus-tunable optofluidic and fast response time of the MBS lenses. It discusses the corresponding surface-based switchable (SBS) technology application for a tristate switchable eyewear lens to provide single focus viewing at three different distances (far, intermediate, and near) under the action of a single actuator with fast response time between foci switching and frameindependent capability.

\section{Materials and Methods}

\subsection{Principle of Operation}

The concept of switching between two different optical states to achieve switching between different foci was applied to the design of the optofluidic element as described by Portney. ${ }^{14}$ Each optical state manifests its own optical power, and switching between the optical states allows for switching between the corresponding optical powers, thus avoiding a continuous change in foci that reduces response time. Thus, the performance of the optofluidic element was digitized by introducing different optical states-refractive and diffractive optical states of different optical powers. As a digitized optofluidic element is combined with a conventional lens, for example, a spectacle lens, the result is a switchable spectacle lens; see Fig. 1. The digitized optofluidic optical element refractive state of a certain power is set by the membrane in the refractive form of a certain curvature, being flat in the simplest refractive form; the diffractive state of a higher power is set by the membrane taking the diffractive form of a relief shape.

The refractive state occurs with the optical fluid filling the space between the membrane and diffractive guiding surface of the optical substrate (active chamber), thereby producing the membrane in the refractive form. The optical fluid is the matching fluid, meaning that its refractive index is very close to the refractive index of the optical substrate material, thus masking the diffractive guiding surface of the optical substrate to produce the optofluidic element in the refractive state of a certain optical power and masking the through-holes. As the matching fluid is removed from the active chamber into the internal chamber via through-holes placed along the thinnest portions of the optical substrate of each diffractive groove, the membrane takes the diffractive form by conforming to the diffractive guiding surface shape; thereby, the optofluidic optical element takes the diffractive state of a different optical power set by the diffractive guiding surface. Non-matching optical fluid occupies the external chamber, providing fluidmembrane-fluid structures to reduce the gravity effect. ${ }^{7,8}$ The result is a self-contained digitized optofluidic element that switches between preset optical powers with the membrane shape changing between the optical forms. This explains the use of the term "surface-based switchable" optical element (SBS OE) to describe the technology. 


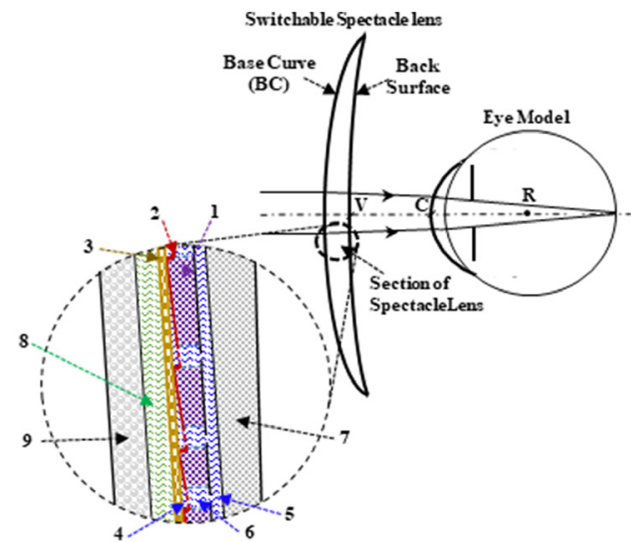

(a)

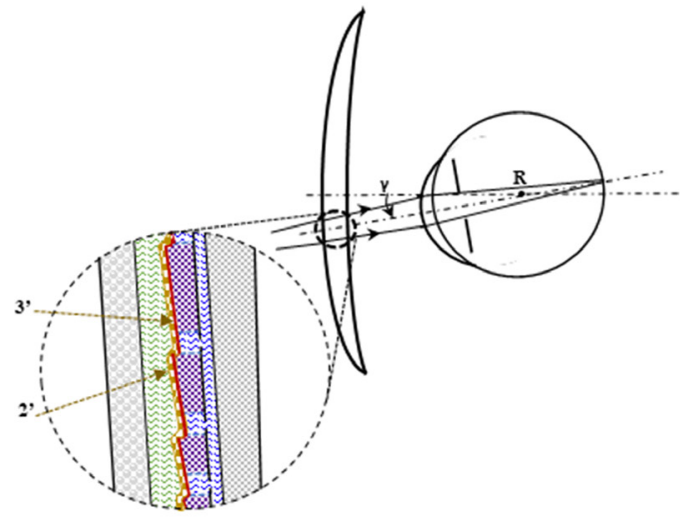

(b)

Fig. 1 A section of a bistate switchable spectacle lens with a digitized optofluidic optical element of a multi-chamber design in the refractive and diffractive states. (a) A switchable lens in the refractive state at a far object viewing. The spectacle lens is placed at the vertex distant VC from the cornea of the eye. In spectacle ray tracing, the $\mathrm{VC}$ is commonly set to $13 \mathrm{~mm}$, and the eye rotation is set at point $R$ at the distance from the front of the eye $C R=14 \mathrm{~mm} .{ }^{15-17}$ A section of the switchable spectacle lens demonstrates the structure of the digitized optofluidic element. There is optical substrate 1 with diffractive guiding surface 2 facing the elastic membrane 3 with space between them, called active chamber 4 , filled with the matching fluid (refractive index matches refractive index of the optical substrate material). An active chamber is connected to internal chamber 5 filled with the matching fluid via the through holes 6 . The internal chamber 5 is formed between the optical substrate 1 and substrate support 7 . The external chamber 8 at the side of the membrane opposite the active chamber is filled with non-matching fluid. It is formed between the membrane 3 and membrane cover 9 . External surfaces of the membrane cover 9 and substrate support 7 form front $\mathrm{BC}$ and back RS of the switchable spectacle lens. (b) The switchable lens in the diffractive state for near viewing with eye rotation for downgaze. An additional volume of non-matching fluid is injected by an actuator into the external chamber 8 to press the membrane 3' against the diffractive guiding surface 2 , thereby transporting a volume of the matching fluid in the active chamber 4 to the internal chamber. The front surface 2' of the membrane takes a diffraction shape that closely resembles the diffraction shape of the diffractive guiding surface to manifest higher optical power for near viewing.

\subsection{Optofluidic Design}

The active chamber of the optofluidic element (Fig. 1) is very shallow, with the groove height being a few tens of microns; $2 \mu \mathrm{l}$ of fluid transfer is required for switching within, for instance, a 15-mm active optic diameter (diameter of the diffractive guiding surface) of the SBS optical element.

Polydimethylsiloxane (PDMS) material was selected for the elastic membrane. Table 1 describes the material characteristics of the membrane in $\mathrm{PDMS}^{18}$ and the optical substrate in polymethylpentene (TPX) ${ }^{19}$ of the SBS optical element.

A selection of optical fluids is critical for the SBS OE operation. Table 2 describes tetraethylene glycol as the matching fluid of the substrate and perfluorodecalin as the non-matching fluid and summarizes their characteristics. ${ }^{20-25}$

Table 1 Materials of key optical parts.

\begin{tabular}{lccccc}
\hline \hline Material (abbreviation) (use) & $\begin{array}{c}\text { Refractive } \\
\text { index }\left(20^{\circ} \mathrm{C}\right)\end{array}$ & Elasticity & Inertness & $\begin{array}{c}\text { Optically } \\
\text { clear }\end{array}$ & Comment/usage \\
\hline PDMS (elastic membrane) & 1.41 & High & Good & Yes & $\begin{array}{c}\text { Pharmaceutical and } \\
\text { medical devices }\end{array}$ \\
PMP or TPX ${ }^{\circledR} /$ (optical substrate) & 1.46 & N/A & Good & Yes & Medical equipment \\
\hline \hline
\end{tabular}


Table 2 Optical fluids of SBS optical element.

\begin{tabular}{lccccccc}
\hline \hline & $\begin{array}{c}\text { Refractive } \\
\text { index } \\
\left(20^{\circ} \mathrm{C}\right)\end{array}$ & $\begin{array}{c}\text { Viscosity } \\
(\mathrm{cP} \text { at } \\
\left.25^{\circ} \mathrm{C}\right)\end{array}$ & $\begin{array}{c}\text { Silicone } \\
\text { membrane } \\
\text { permeability }\end{array}$ & Inertness & $\begin{array}{c}\text { Bio- } \\
\text { compatibility }\end{array}$ & $\begin{array}{c}\text { Optically } \\
\text { clear }\end{array}$ & $\begin{array}{c}\text { Comment/ } \\
\text { usage }\end{array}$ \\
\hline $\begin{array}{l}\text { Optical fluids [use] } \\
\text { (non-matching) }\end{array}$ & 1.3145 & 2.94 & Low & Good & Yes & High & $\begin{array}{c}\text { Eye retinal } \\
\text { procedures }\end{array}$ \\
$\begin{array}{l}\text { Tetraethylene } \\
\text { glycol (matching) }\end{array}$ & 1.459 & 58.3 & Low & Hygroscopic & Okay & High & $\begin{array}{l}\text { Fragrance } \\
\text { ingredient }\end{array}$ \\
\hline \hline
\end{tabular}

Lee et al. ${ }^{20}$ described the results of extensive experiments on fluid solubility with PDMS. The author showed swelling ratios for PDMS soaked in fluids representing a variety of polarities. We were interested in two of these fluids, both of which exhibited a swelling ratio of 1, i.e., no swell. These fluids were perfluorodecalin (the non-matching fluid) and ethylene glycol, which is related to our matching fluid, tetraethylene glycol.

Key characteristics of the selected optical fluids are (1) PDMS is impermeable to matching and non-matching fluids, (2) the matching fluid refractive index matches the refractive index of the optical substrate material (1.46), and (3) matching and non-matching fluids manifest a significant difference in refractive indices to allow for lowering a guiding surface groove height and, therefore, transferring of only a tiny amount of optical fluid in and out of the active chamber for switching between the optical states.

\subsection{Membrane Mechanical Characteristics Analysis}

Final element analysis (FEA) was applied to analyze the PDMS membrane conformance to the guiding diffractive surface ${ }^{26}$ (Innova, Irvine, California). The primary goal of FEA was to establish membrane elastic characteristics for conformance to the diffractive guiding surface of the relief shape for different groove heights of 6,8 , and $16 \mu \mathrm{m}$ were used.

Figure 2 shows an example of the membrane conformance [Fig. 2(a)] and conformance curves (CCs) for different groove heights [Fig. 2(b)] defined as a function of the membrane conformance to a groove of the diffractive guiding surface with load. Load is defined as a difference in pressures between external and internal chambers to produce membrane conformance to the groove.

As shown in Fig. 2(a), a diffractive groove commonly includes groove smoothing by the cutting tool, which is acceptable due to masking by the matching fluid. The membrane manifests own membrane smoothing at a groove transition due to the membrane continuous shape. The membrane smoothing width is $S=L-L^{\prime}$.

At certain conditions, the CCs rapidly increase with load up to a certain zone and then continue at a slower pace; a transition point (TP) can be selected at such a zone as the point between the fast and slow paces. A good TP quantification of smoothing $\mathrm{S}$ is where the pace changes is $S=2 H$ (double of groove height). A good fitting of such curves is shown as

$$
y=\frac{x}{a+b x+c x^{2}+d \sqrt{x}}
$$

where $y=$ conformance and $x=$ load (PSI). An example of the parameters of $8-\mu \mathrm{m}$ groove height CC [Fig. 2(b)] is $a=-0.01 ; b=0.82 ; c=0.02 ; d=0.4$

In this regard, the FEA has revealed that, as long as the ratio (groove width $L$ )/(groove height $H$ ) $>10$ (Rule of 10), the TP value is pronounced on the CC [groove heights 6 and $8 \mu \mathrm{m}$ of Fig. 2(b), for instance] as the transition between the fast and slow paces at the curve. Another recommendation by FEA was the benefit of truncating and rounding the diffractive surface peaks to avoid membrane damage and minimize strain.

In designing a switchable optofluidic element consisting of many grooves, only the smallest groove CC is analyzed for switching between the optical states. Such a groove must meet the "Rule of 10"; therefore, switching pressure can be placed at the TP to manifest high conformance 


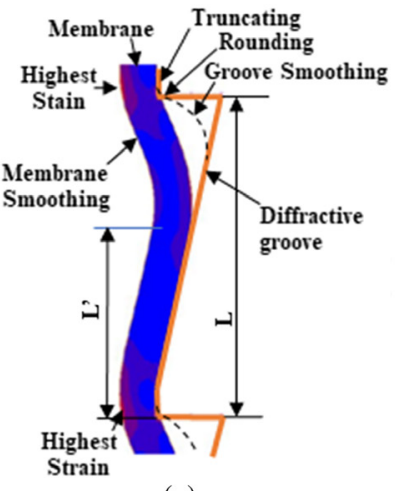

(a)

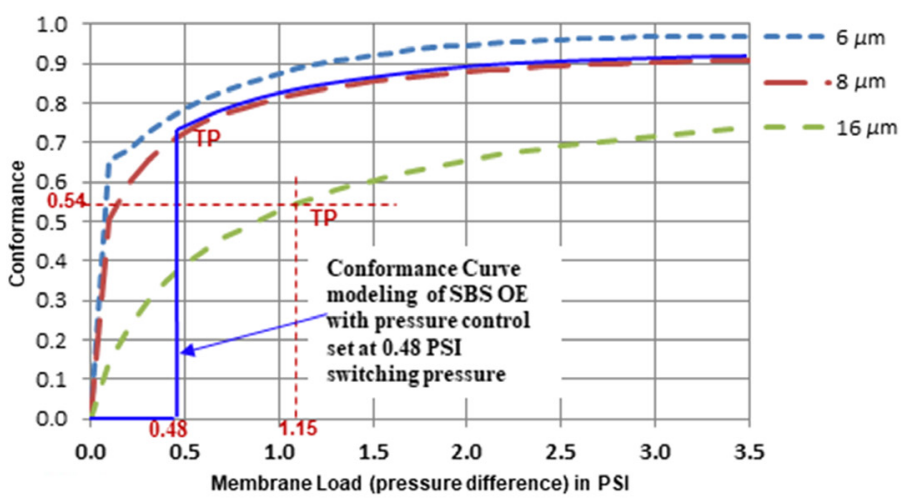

(b)

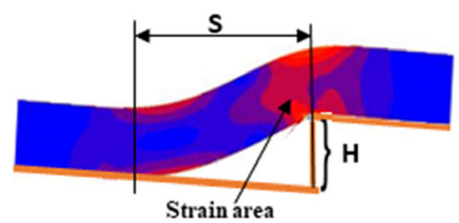

(c)

Fig. 2 Membrane conformance to a diffractive surface groove and CCs. (a) Deformation of 10- $\mu \mathrm{m}$ thickness PDMS membrane over the diffractive surface groove of the width $L=88 \mu \mathrm{m}$ and height $H=16 \mu \mathrm{m}$, and the locations of the largest strain shown in red. The membrane conformance to the groove was within width $L^{\prime}$ and the conformance measure defined as $C=L^{\prime} / L$ was $C=0.54$ for the load of $1.15 \mathrm{PSI}$. (b) CCs. The CC in green (lowest one) references the membrane of (a) shown with the conformance at a point selected as the TP with membrane smoothing $S=L-L^{\prime}=2 H$, where $H$ is groove height. CCs for 8- and 6- $\mu \mathrm{m}$ groove heights demonstrate slow to fast paces occurring if the groove width is more than 10 times groove height (Rule of 10). (b) The CC (solid blue) with pressure control set at $0.48 \mathrm{PSI}$ for switching between the refractive and diffractive forms of the membrane. The corresponding CC is a modification of the CC (red) for $8-\mu \mathrm{m}$ groove height with the inclusion of a pressure control. (c) $20-\mu \mathrm{m}$ membrane deformation showing a larger strain for similar membrane smoothing $S$ to $10-\mu \mathrm{m}$ membrane in (a).

and, therefore, diffraction efficiency (DE) in the diffractive state. Non-conformance (smoothing $\mathrm{S}$ ) is maintained for all other grooves because of the consistency in load for the same groove height and membrane of constant thickness. It results in higher conformance of the rest of the grooves because they are wider sizes and, therefore, manifests higher diffraction efficiencies than the smallest width groove. Thus, by designing the diffractive guiding surface with the appropriate smallest groove width, we ensure that a switchable optofluidic element manifests high DE in the diffraction state.

FEA also indicated the benefit of including in the SBS OE design a pressure control element to set the pressure at which switching between the optical states occurs, that is switching pressure $\mathrm{P}_{\mathrm{S}}$. It is also desirable to set a switching pressure $P_{S} \geq P_{\mathrm{TP}}$, where $P_{\mathrm{TP}}$ is a load at the TP of the $\mathrm{CC}$ of the membrane at the narrowest groove of the diffractive guiding surface. Such a selection ensures that switching between the optical states occurs simultaneously at all diffractive grooves of the diffractive guiding surface. This also ensures that the membrane refractive form is maintained up to a switching pressure $P_{S}$.

\subsection{Diffractive State Optical Quality Evaluation}

Conformance analysis in the prior section demonstrated the presence of the membrane nonconformance in the diffractive state. For instance, Figs. 2(a) and 2(c) show that the membrane at the membrane smoothness area is formed by a small radius. The membrane smoothing reduces DE at each groove, and the small radius shape creates a spread-out background luminance at the image formed in the diffractive state, which may result in ocular straylight to reduce image contrast. 
The task was to assess the effect of different levels of light background on the image contrast. The modeling was conducted to determine the effect of out-of-focus blur simulating an ocular straylight on the modulation transfer function (MTF) of the in-focus image. The Zemax Optical Design Software (Zemax, LLC, Kirkland, Washington) was used for calculations for the eye model $^{27}$ and spectacle lens ${ }^{15-17}$ combination, with the spectacle lens being a multi-configuration setup with one configuration for the zero-order image and another for the first-order image. In such modeling, the "switchable" element, consisting of fluids and optical substrate of the materials used in prototyping and switchable optical element design, was placed within the spectacle lens as shown in Fig. 1.

The MTF evaluation involved the use of a zero-order configuration to create a blur to emulate optical straylight and a first-order configuration to form the image in the diffractive state. The combined configurations produce the overlap of the blur and in-focus spot diagrams for Huygens MTF calculation; see Fig. 3 .

The modeling involved taking MTF of the spectacle lens + eye model with both 0-order (object at infinity) and first-order (object at near) at best foci at the retina (image plane), thus creating an optical condition analogous to the switchable element operation. The spectacle lens consists of the base curve (BC) at the front surface and refractive surface (RS) to form $-4 \mathrm{D}$ refraction. The diameter of the switchable optical element was set to $15 \mathrm{~mm}$, and the optimization for MTF was conducted for rotation within this diameter minus $1.5 \mathrm{~mm}$ to avoid vignetting at a $3-\mathrm{mm}$ aperture. The resulting eye rotation is up to about $12 \mathrm{deg}$. This MTF of the first-order image was taken with $100 \%$ of rays allocated to become the Reference MTF. Then the shape of the back surface of the spectacle lens was changed to $\mathrm{RS}_{\mathrm{S}}$ to create a significant blur in the zero-order configuration. Membrane smoothing likely creates a significant light spread, and the surface $\mathrm{RS}_{\mathrm{S}}$ was changed as much as possible but still allowed for optimization of the MTF of the first-order to the level of Reference MTF. The optimization was conducted for $r^{2}, r^{4}$, and $r^{6}$ phase terms by the same merit function used for the Reference MTF. The setting now permitted the application of different diffraction efficiencies to the first-order by splitting the number of rays between two configurations and calculating the corresponding Huygens MTFs; Fig. 3(c) shows the MTFs at $100 \%, 85 \%$, and $50 \%$ diffraction efficiencies.

Next, the DE of the switchable lens in the diffractive state was determined. The process involved setting up the same membrane smoothing width to the grooves. The switching of the design was defined for smoothing $S=2 \cdot H$, where $H=22.3 \mu \mathrm{m}$ (Sec. 2.4). A DE of the diffractive state for a diffractive guiding surface of ( $n$ ) grooves was determined per Eq. (2) for eye model axial position:

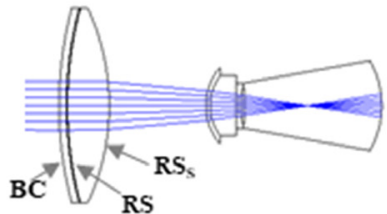

(a)

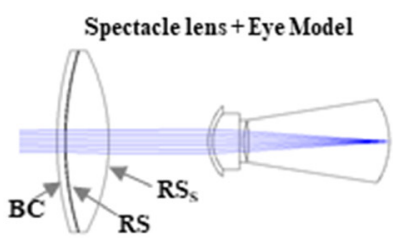

(b)

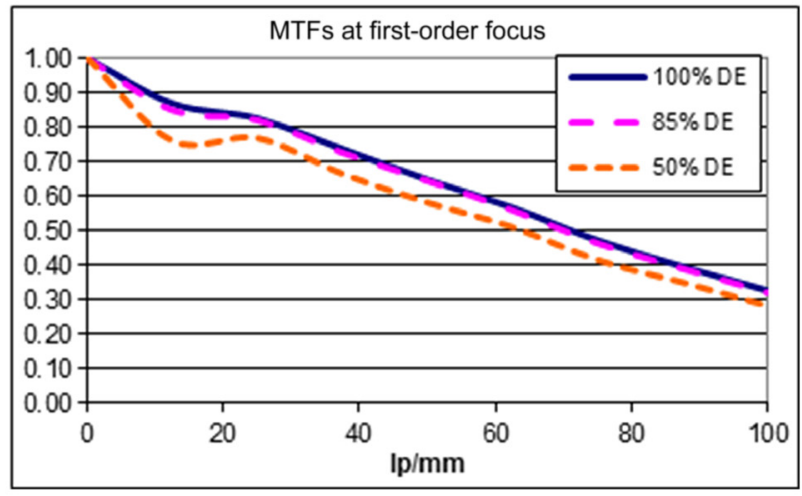

(c)

Fig. 3 Modeling of the optical performance of the switchable spectacle lens in the diffractive state. (a) Blur formed by the system of Spectacle Lens and Eye Model in zero-order configuration as emulation of ocular straylight. (b) Focusing in first order configuration. (c) Huygens MTFs at different light splits between zero-order and first-order configurations, which is equivalent to diffraction efficiencies at first-order diffraction state. MTFs at DE $=100 \%, 85 \%$, and $50 \%$ represent conditions where $0 \%, 15 \%$, and $50 \%$ fractions of light, respectively, are allocated to membrane smoothing at the smallest width groove per Eq. (2b) with $p=6$ and $F_{d}=500 \mathrm{~mm}$ (2D power switching). 


$$
D E=\sum_{i=1}^{n}\left[\frac{\left(r_{i}-2 H\right)^{2}-r_{i-1}^{2}}{r_{n}^{2}}\right]
$$

Per Eq. (2b), $p=6$ and $F_{d}=500 \mathrm{~mm}$ (2D power switching is addressed in Sec. 4), and only two central grooves are exposed at $5-\mathrm{mm}$ diameter, resulting in $\mathrm{DE} \approx 0.94$. With a 12 deg eye rotation, the DE was determined by the groove crossing the center of the aperture projected onto the spectacle lens. Per Eq. (2), $p=6$ and $F_{d}=500 \mathrm{~mm}$, and it occurs with the $r_{11}$ groove, resulting in $\mathrm{DE} \approx 0.84$. The lowest $\mathrm{DE}$ occurred for the peripheral viewing. According to the MTFs of Fig. 3(c), the effect of blur on the MTF simulating membrane smoothing effect on contrast would be noticeable at DE below $\approx 0.5$ (about $5 \%$ of MTF reduction); therefore, DE $=$ 0.84 has a very minimal effect on image quality in the diffractive state.

\subsection{Prototyping}

A SBS optical element prototype was made for the "proof-of-concept" to study the membrane shape change between the refractive and diffractive forms in the multi-chamber design; see Fig. 4.

Optical substrates of $100 \mu \mathrm{m}$ thickness were made from TPX with a diffraction zone diameter of $15.432 \mathrm{~mm}$. To have a manageable number of grooves, a multimode design with a phase depth of $p=6$ was used. ${ }^{28,29}$ The corresponding groove height $H_{p}$, radii of grooves $r_{j}$ where $j$ (groove numbers) $=1,2, \ldots$, and wavelength peaks $\lambda_{\text {peak }}$ of the DE are defined by Eqs. (2a)-(2c). The design wavelength $\lambda_{d}=0.55 \mu \mathrm{m}$ corresponds to the peak of the eye photopic color sensitivity, $m=$ diffraction order, and refractive indices of the optical substrate $(n)$ and non-matching fluid $\left(n^{\prime}\right)$ are listed in Tables 1 and 2.

$$
\begin{gathered}
H_{P}=\frac{p \lambda_{d}}{n\left(\lambda_{d}\right)-n^{\prime}\left(\lambda_{d}\right)}, \\
r_{j}^{2}=2 j p \lambda_{d} F_{d},
\end{gathered}
$$

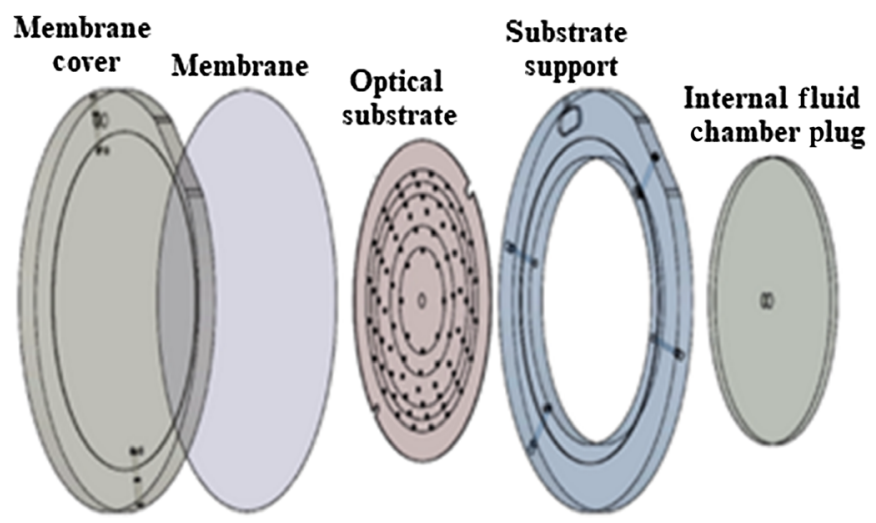

Fig. 4 Prototype of a bistate SBS optical element containing one elastic membrane. It demonstrates the prototype parts that include "substrate support" (PMMA material) with four side channels, the "chamber plug" (PMMA material) to create an internal chamber with the "optical substrate" and "substrate support." The chamber plug also supports the thin "optical substrate" (TPX material) by the central post. The "optical substrate" includes multiple through-holes at each diffractive groove. The "membrane" (PDMS material) is adjacent to the "optical substrate" to form an active chamber between that is filled with the matching fluid via substrate support's two side channels. Another two side channels of the substrate support are used to fill the internal chamber with the matching fluid. The "membrane cover" (PMMA material) forms an external chamber with the membrane, which is filled with the non-matching fluid via the membrane support's two side channels. 
Portney, Christ, and Christ: Digitized optofluidic element and its application to tristate lens...

Table 3 Diffractive guiding surface and through holes.

\begin{tabular}{|c|c|c|c|}
\hline Groove No. & Groove radius (mm) & Groove width $(\mathrm{mm})$ & Holes no per groove \\
\hline 1 & 3.146 & & 8 \\
\hline 2 & 4.450 & 1.304 & 11 \\
\hline 3 & 5.450 & 1.000 & 14 \\
\hline 4 & 6.293 & 0.843 & 16 \\
\hline 5 & 7.036 & 0.743 & 18 \\
\hline 6 & 7.707 & 0.671 & 20 \\
\hline
\end{tabular}

The height of the diffractive grooves was $H_{6}=22.68 \mu \mathrm{m}$. The peaks of the diffractive groove were truncated by $0.375 \mu \mathrm{m}$ and rounded during lathe cutting to avoid membrane damage and to minimize strain; the final groove height was $22.30 \mu \mathrm{m}$. The total number of 87 through-holes of $0.300-\mathrm{mm}$ diameters were micro drilled through the optical substrate's thinnest portions next to the groove's transitions and were distributed over each groove with equal spacing. Table 3 lists the dimensions of the grooves for the focal length $F_{d}=1500 \mathrm{~mm}$ and the through-holes distribution per groove. The focal length value was selected for the prototyping to incorporate micro-drilling in the through-holes fabrication.

PDMS membrane sheets of 20- $\mu$ m thickness with Mylar carrier (to maintain membrane integrity) were purchased under the tradename Silpuran $^{\mathrm{R}}$ (Wacker Chemie AG, Munich, Germany) and sized for the prototyping. The remaining prototype parts were made of polymethyl methacrylate (PMMA).

Three processes were applied during prototype assembly to investigate their capabilities: (a) TPX-PMMA solvent with primer bonding, (b) PDMS to PMMA by surface functionalization and oxygen plasma bonding, ${ }^{30-32}$ and (c) PMMA-PMMA simple solvent-based bonding. ${ }^{33}$

Complete digitized optofluidic prototypes were assembled to analyze the fabrication processes and were filled with the fluids. To evaluate membrane conformance, the prototypes without a membrane cover attached, so called "incomplete" samples, were used. The side channels were used to fill in the active and internal chambers with blue dyed matching fluid (tetraethylene glycol). One channel connected to the internal chamber was left connected to 100-ml syringe to serve as the actuator and the other three channels were blocked.

Bubbles were one of the most difficult aspects of the assembly, and the assembly process was redesigned to change from assembling the chambers and then filling them with the fluids to filling the spaces with the fluids and then forming the chambers, each filled with the corresponding fluid. For this purpose, the membrane is bonded within a ring-shaped structure to expose both sides of the membrane. For instance, to create the active chamber filled with the matching fluid, the appropriate side of the ring-shape structure with exposed membrane is filled with the matching fluid, and then the optical substrate is adhered to the internal side of the ring-shaped structure, creating the active chamber filled with the matching fluid. An excess of the matching fluid is removed via the through-holes to the opposite side of the optical substrate and is used to create the internal chamber filled with the matching fluid by adhering the substrate support to the same internal side of the ring-shale structure. An excess of matching fluid is removed via an appropriately placed opening, which is then blocked. Similarly, an external chamber filled with the non-matching fluid is created at the opposite side of the membrane.

\subsection{Switching Response Time}

How fast the switching between the optical states occurs also depends on a fluid flowrate at the through-holes of the multi-chamber configuration. The question can be addressed with the help of the Bernoulli equation: ${ }^{34}$ 


$$
P+\frac{1}{2} \rho \cdot V^{2}+\rho \cdot g \cdot h=\text { constant }
$$

where $P=$ pressure, $\rho=$ density, $V=$ volume, $h=$ elevation, and $g=$ gravitational acceleration.

The Bernoulli equation is the approximation of analyzing a fluid flow along a streamline between two locations with several assumptions, i.e., the incompressibility, steady flow requirement (flowrate does not change in time), and no viscosity. The matching fluid (tetraethylene glycol) is incompressible, but it is unsteady; flowrate is time dependent. This involves additional pressure to accelerate; therefore, the Bernoulli equation is an approximation in this application. The matching fluid also manifests viscosity, which can be ignore in a very short channel; therefore, we assess a response time without taking into account matching fluid viscosity.

In the present application, the elevation is not considered, and Eq. (3a) is reduced to Eq. (3b), which shows a flowrate dependance on a difference in pressure.

$$
P_{1}-P_{2}=\frac{1}{2} \cdot \rho \cdot\left(V_{2}^{2}-V_{1}^{2}\right)
$$

Using the Bernoulli equation, for the matching fluid mass density of $1.009 \mathrm{~g} / \mathrm{ml}$ and throughhole diameter of $0.3 \mathrm{~mm}$, the flowrate was $Q_{\min }=0.13 \mathrm{ml} / \mathrm{s}$, for a difference in pressure between the chambers $\Delta P=0.25$ PSI. The flowrate can be increased with the increase in the difference in pressure, for instance, the flowrate is doubled to $0.26 \mathrm{ml} / \mathrm{s}$ for $\Delta P=1$ PSI. Even for $25 \mathrm{~mm}$ of active optic diameter, the transferred volume is tiny, only $\approx 11 \mu 1$ per two-state switching. The transfer volume per through-hole $V_{\mathrm{H}} \approx 0.08 \mu \mathrm{l}$ at the second groove (worst case) results in a maximum flow time $t_{F}=V_{H} / Q_{\min }=0.00008(\mathrm{ml}) / 0.13(\mathrm{ml} / \mathrm{s})=0.00062 \mathrm{~s}$. Even doubling the flow time by taking into account acceleration due to unsteady fluid flow still leads to an almost instant fluid transfer between the active and internal chambers. This is due to the uniqueness of the digitized optofluidic element operation that requires only a tiny amount of fluid transfer for switching between the optical states.

Let us consider the effect of viscosity of the matching fluid $\eta=53.8 \mathrm{cP}$. In the case of a cylindrical channel of length $L$, radius $R$, presence of viscosity $\eta$, and the pressure difference $\Delta p_{\eta}$ required to balance viscous resistance in the flow $Q$, we can apply Poiseuille equation, ${ }^{35}$ which is given as

$$
\Delta p_{\eta}=\frac{8 L \eta}{\pi R^{4}} Q
$$

Using a switchable element diameter $15.414 \mathrm{~mm}$ and groove height $0.0223 \mathrm{~mm}$, the fluid volume is determined. Our goal is to maintain the pressure below 2 PSI for piezo-electrical actuation and not to exceed a $0.2 \mathrm{~s}$ response time. Applying a through-hole length of $L=0.05 \mathrm{~mm}$, diameter $0.30 \mathrm{~mm}$, and 20 though-holes per groove, the $\Delta p_{\eta}=1.8$ PSI. Thus, the viscosity appears to be the dominant factor effecting pressure and controlling the response time in a surface-based switchable optical element.

\section{Results}

Figure 5 shows elastic membrane shape change with matching fluid transfer at the incomplete sample (the samples without the membrane cover attached).

Figure 5(b) shows a photo of an incomplete sample with membrane in the refractive form with matching fluid (with blue dye) filling the active chamber between the membrane and substrate. Figure 5(c) shows a photo of the same sample with the membrane in the diffractive form created by the matching fluid removal from the active chamber to the internal chamber of the sample. The shape and size of the grooves of the membrane in Fig. 5(c) were remarkably similar to those of the diffractive guiding surface of the sample in Fig. 5(a). Figure 5(d) confirms similarities by a close line-up of the membrane's surface profile in the diffractive form and the profile of the guiding diffractive surface in terms of groove widths and their slopes. 


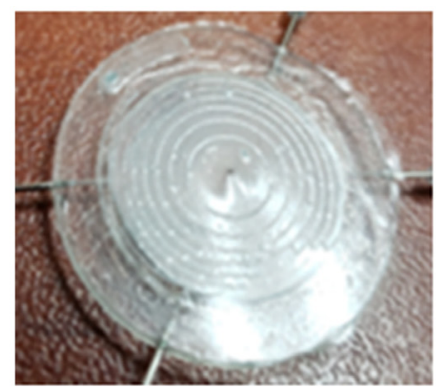

(a)

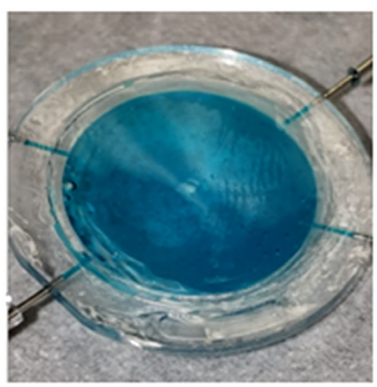

(b)

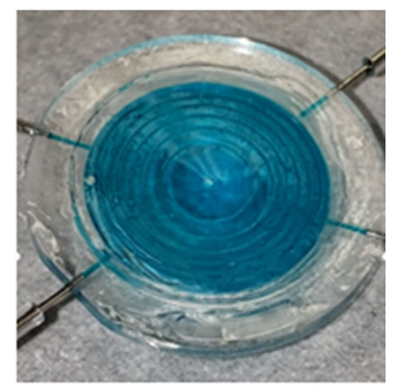

(c)

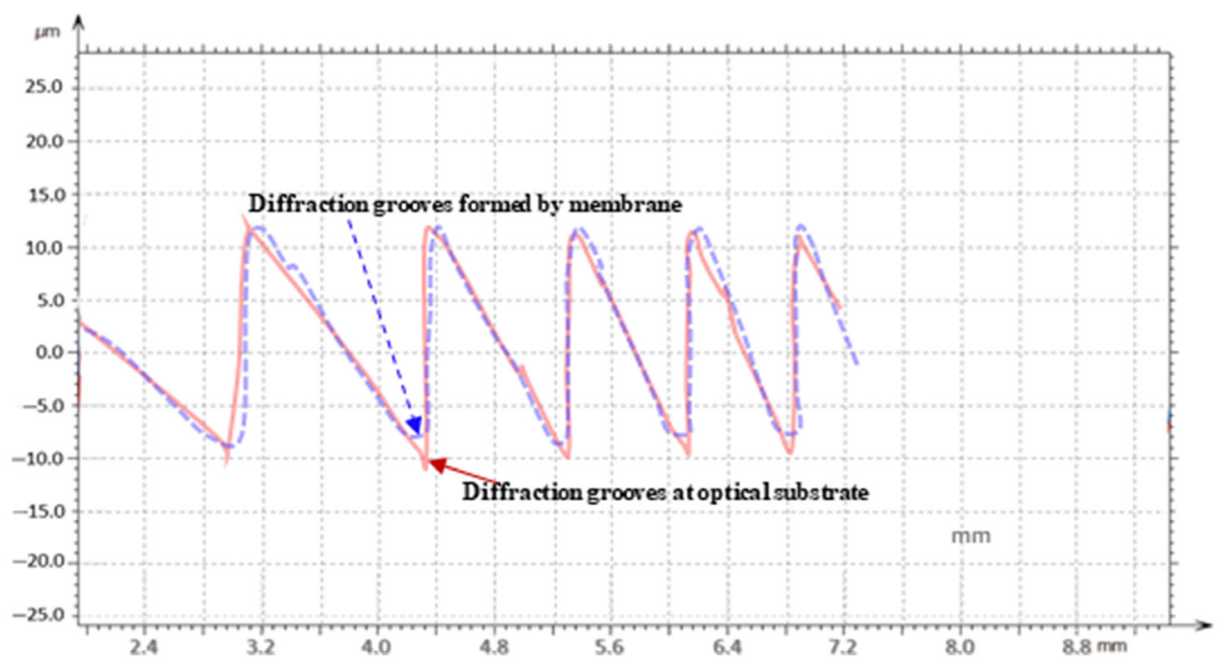

(d)

Fig. 5 Demonstration of membrane conformance to diffractive guiding surface for the incomplete prototype sample. (a) A photo of an incomplete sample without matching fluid. (b) A photo of an incomplete sample filled with blue dyed matching fluid and membrane in the refractive form. (c) The photo of the same incomplete sample with membrane in diffractive form. (d) Data taken by Nanovea HS2000 Profilometer comparing groove shapes of the optical substrates (solid red) and membrane surface in the diffractive form of the (c).

\section{Discussion}

The advantage of the SBS optical element over the MBS element is not only the simplicity of its switching operation but also the option to expand the bistate operation of Fig. 1 (switching between two foci) to a tristate operation (switching between three foci) with the use of a single actuator. A tristate configuration is shown in Fig. 6.

The three-state optofluidic design includes two assemblies, each consisting of a membrane and optical substrate with the corresponding active chamber connected via through-holes to the internal chamber, both filled with the matching fluid. A non-matching fluid fills the single external chamber between the membranes, which is used for a switching control by a single actuator. A switching actuation of each assembly (membrane shape switching between the refractive and diffractive forms) occurs by the actuator chamber interaction with the external chamber through the volume change. The three-state design is particularly beneficial for eyewear as the switching occurs between the optical power for far vision $\left(P_{\text {far }}\right)$ with both membranes being in the refractive form [Fig. 6(b)], an optical power for intermediate vision $\left(P_{\text {intermediate }}\right)$ in which one of the membranes is in the diffractive form [Fig. 6(c)], and an optical power for near vision $\left(P_{\text {near }}\right)$ in which both membranes are in the diffractive forms [Fig. 6(d)].

A change between the refractive and diffractive forms of each membrane occurs at different switching pressures of two pressure controls set at different switching pressure levels [Fig. 6(a)]. As the pressure in the external and internal chambers reaches a switching pressure of one of the pressure controls, for instance, pressure control 2 , it compresses to allow the matching fluid to 


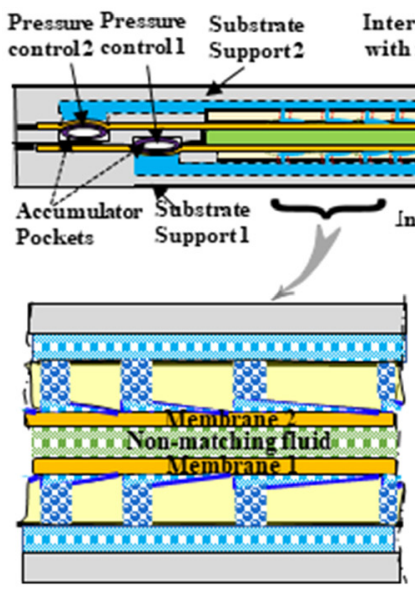

(b)

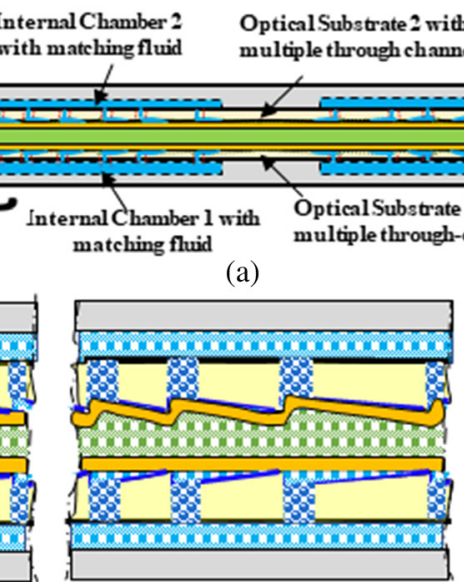

(c)
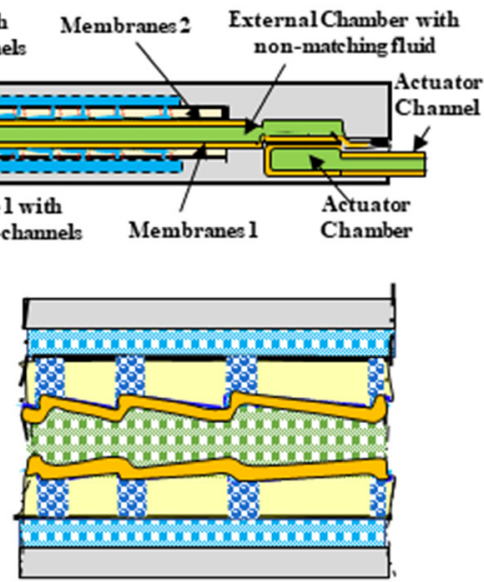

(d)

Fig. 6 Tristate SBS optical element. (a) A cross-section of the tristate optofluidic element and activation chamber connected to an actuator (not shown) via the actuator channel. The actuation chamber interacts with the external chamber in foci switching. Other elements (membrane, active chamber, optical substrate, and internal chamber) are doubled. (b) A small section of (a) with both membranes in the refractive form. (c) The same small section with one of the membranes in the diffractive form and another membrane maintaining the refractive form. (d) The same small section with both membranes in the diffractive form.

transfer from the active chamber 2 to the connected internal chamber 2 with the membrane 2 taking the diffractive form of the guiding diffractive surface of the optical substrate 2 [Fig. 6(c)]. As the pressure in the external chamber increases further with the actuation, it reaches a switching pressure of the pressure control 1 , which results in the membrane 1 taking the diffractive form of the guiding diffractive surface of the optical substrate 1 [Fig. 6(d)].

In far viewing, the switchable spectacle lens is in the refractive state similar to conventional spectacles and manifests positive or negative $\mathrm{LCA}_{\mathrm{R}}$ depending upon the positive or negative spectacle lens power. Its value is determined by the materials dispersion and can be an addition to or deduction from eye $\mathrm{LCA}_{\mathrm{E}}$. In any case, it should not present an issue for far viewing in the refractive state based upon experience with a conventional spectacle lens. In near viewing, the switchable spectacle lens is in the diffractive state of a positive Add power resulting in negative $\mathrm{LCA}_{\mathrm{D}}$. In appraising $\mathrm{LCA}_{\mathrm{D}}$, we can take a cue from the extensive experience with diffractive intraocular lenses. ${ }^{36}$ Due to a small Add power value (up to $4 \mathrm{D}$ ), the $\mathrm{LCA}_{\mathrm{D}}$ effect on near vision is expected to be small.

Transverse chromatic aberration (TCA) usually presents a bigger issue in spectacle lenses when viewing through the spectacle lens periphery. ${ }^{16,17}$ A diameter of the switchable optical element within the switchable spectacle lens is limited to an approximately $15 \mathrm{~mm}$ diameter, thus minimizing the effect of the TCA in the diffractive state $\left(\mathrm{TCA}_{\mathrm{D}}\right)$ in near viewing.

A multimodal phase depth of $p=6$ was used in the prototyping and can be applied to a digitized optofluidic element in eyewear application. It not only allows for the formation of a manageable number of grooves but also improves the chromatic characteristics of the design by creating at least three peaks of diffractive efficiency within the visual light spectrum range. ${ }^{28,29}$ For instance, according to Eq. (2c), DE reaches $100 \%$ at three wavelengths $\lambda_{\text {peak }}$ : at red $(0.66 \mu \mathrm{m})$ at the diffractive order $m=5$, green $(0.55 \mu \mathrm{m})$ at $m=6$, and blue $(0.47 \mu \mathrm{m})$ at $m=7$ for the phase depth $p=6$. It is worth noting that a multimodal diffractive design has been successfully used in optometry in the Noves spectacles for low vision applications ${ }^{37}$ (Eschenback Optik GmbH, Germany).

The tiny amount of fluid transfer involved in switching between foci allows for a very compact overall design: (1) the three-state optofluidic element may reach $\sim 300 \mu \mathrm{m}$ thickness and (2) a micro actuator and its driver of small sizes with a low power consumption can be applied. As an example, the actuation by a piezo-electric bender is a well-established technology; ${ }^{38}$ a commercially available piezo-electric bender T10 by Johnson Matthey Piezo Products GmbH, 
Germany, was considered. Its maximum blocking force is $0.13 \mathrm{~N}$ at $130 \mathrm{~V}$ driving voltage and its maximum deflection is $\pm 70 \mu \mathrm{m}$. The operational specs are the following: unit dimensions are $9(6.5$ active $) \times 1 \times 0.5 \mathrm{~mm}^{3}$ (activator area $A_{a}=6.5 \mathrm{~mm}^{2}$ ) with a deflection of $50 \mu \mathrm{m}$ at $130 \mathrm{~V}$ with blocking force $F_{b}=0.1 \mathrm{~N}$. The maximum pressure is $P_{b}=F_{\mathrm{b}} / \mathrm{A}_{\mathrm{a}} \approx 2.2$ PSI. The fluid volume at the active chamber of the SBS OE design of $15-\mathrm{mm}$ diameter is $2 \mu \mathrm{l}$ in the bistate operation. To transfer such a fluid volume, 13 units of T10 model are needed with the total actuator area of $13 \times 9 \mathrm{~mm}^{2}$. The pressure created by the actuator is adequate for matching fluid transfer with viscosity consideration. The T10 actuator can be driven by the PDu100 Micro Piezo Driver (PiezoDrive, Australia), which is only $12 \times 13 \mathrm{~mm}(0.5 \times 0.5 \mathrm{in})$ and has a supply voltage of $3 \mathrm{v}-5.5 \mathrm{~V}$ supported by a microbattery/photocell combination.

Eyewear for presbyopia correction represents a significant application of the digitized optofluidic element. Targeting 15-mm diameter of the switchable zone (active optical diameter), the bistate SBS OE of the described design is limited to 2D of optical power (focal length $F_{d}=500 \mathrm{~mm}$ ), which covers a significant range of presbyopia correction needs. ${ }^{1}$ A $15 \mathrm{~mm}$ active optical diameter requires 17 grooves: $r_{17}=7.5 \mathrm{~mm}$ per Eq. (2b). The smallest width of the grooves is $\left(r_{17}-r_{16}\right)=0.224 \mathrm{~mm}$, thus meeting the "Rule of 10 " for groove height $H=22.3 \mu \mathrm{m}$, and through-holes of $0.150 \mathrm{~mm}$ are producible by laser drilling or during injection molding.

A fluid volume transfer for a 15-mm active optical diameter of the tristate SBS OE is double the bistate operation, i.e., $4 \mu \mathrm{l}$ ( $2 \mu \mathrm{l}$ fluid volume transfer for switching between two states). The tristate SBS OE provides up to 4D Add power, thus covering the full range of presbyopia correction needs. A custom design actuator is under consideration to transfer the fluid volume in tristate operation with an adequately compact actuator area.

Thus, the tiny volume transfer in the digitized optofluidic element operation leads to compact actuation components and makes it feasible to place them within the switchable eyewear lens itself without involving a spectacle frame, i.e., the potential for frame independence. Figure 7

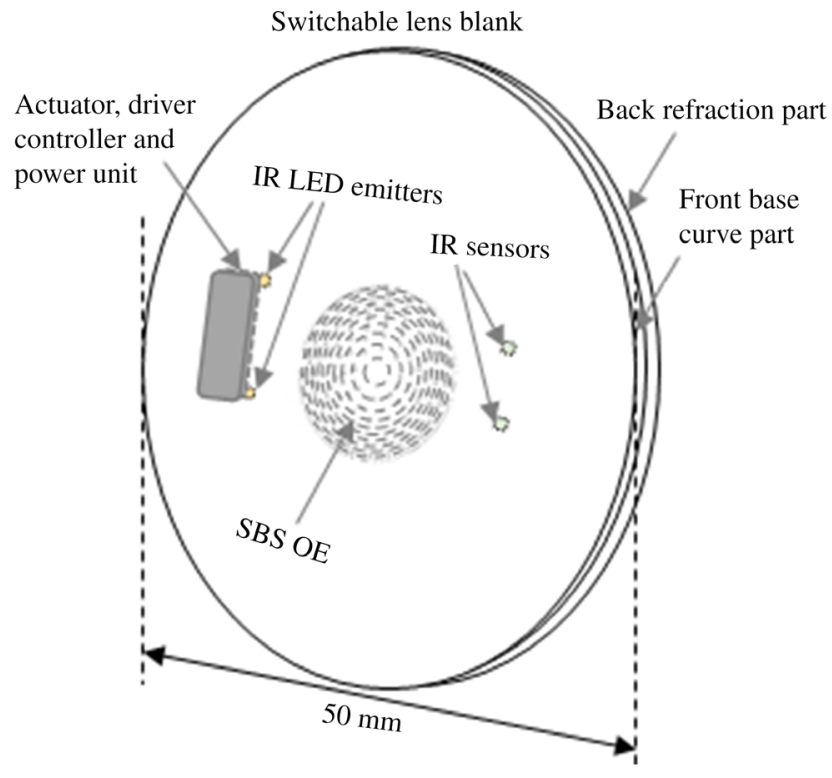

Fig. 7 Modeling switchable lens blank. The bistate (tristate) SBS optical element together with Operational Unit that includes Actuation Unit (actuator, driver, controller and power unit), lightemitting diode emitters and infra-red sensors is embedded between back refraction (BR) part (sphero-cylinder error correction) and Front BC part. BC part is of a constant shape within each refraction increment, and multiple lens blanks are supplied to cover the full range of refractions. ${ }^{16,17}$ BR surface is cut to the required sphero-cylinder shape for an individual refraction during dispensing. The Actuation Unit may protrude at the front of the BC part. Emitters and sensors are known to be used for eye tracking ${ }^{39}$ and suitable for triggering a switching between foci by tracking binocular convergence and/or each eye gaze. 
shows an example of a model of a switchable lens blank for switchable spectacles for presbyopia correction.

The frame independence of a switchable lens blank would be suitable for conventional spectacles dispensing; switchable lens blanks are provided to an optometry lab for switchable eyewear dispensing similar to conventional single focus spectacles dispensing. ${ }^{40}$

\section{Conclusion}

This paper has introduced the digitized optofluidic element of the multi-chamber design and prototyping for the fabrication process evaluation and proof-of-concept demonstration. The digitized optofluidic optic may serve in many applications in which fast switching between predetermined foci is required, and as a significant example, an application to tristate eyewear for presbyopia correction has been discussed.

\section{Acknowledgments}

The authors acknowledge and appreciate the support of Nicholas J. Casella for assisting with prototype testing and conducting silicone-acrylic plasma bonding with surface functionalization. V. Portney is the owner of Vision Advancement LLC and has financial and proprietary interest in surface based switchable technology. F.R. Christ and M.D. Christ are owners of InVision Biomedical, Inc. and have financial interest in surface based switchable technology.

\section{References}

1. "Presbyopia: overview," https://www.ncbi.nlm.nih.gov/books/NBK423833/ (accessed 23 April 2021).

2. N. A. A. Najmee et al., "Satisfaction level of progressive additional lens (PAL) wearers," Enviro.-Behav. Proc. J. 2, 373-382 (2017).

3. A. Selenow et al., "Assessing visual performance with progressive addition lenses," Optom. Vis. Sci. 79, 502-505 (2002).

4. S. R. Lord, J. Dayhew, and J. A. Howland, "Multifocal glasses impair edge-contrast sensitivity and depth perception and increase the risk of falls in older people," J. Am. Geriatr. Soc. 50, 1760-1766 (2002).

5. R. A. Newton, "Balance and falls among older people," Gener. J. Am. Soc. Aging 27, 27-31 (2003).

6. H. Radhakrishnan, W. Neil, and W. N. Charman, "Optical characteristics of alvarez variablepower spectacles," Ophthalmic Physiol. Opt. 37, 284-296 (2017).

7. L. Wang, H. Oku, and M. Ishikawa, "Variable-focus lens with $30 \mathrm{~mm}$ optical aperture based on liquid-membrane-liquid structure," Appl. Phys. Lett. 102, 131111 (2013).

8. Y. Lo and D. Zhang, "Fluidic adaptive lens," WIPO patent WO/2006/011937 (2006).

9. S. Kuiper and B. H. W. Hendriks, "Variable focus spectacles," WIPO patent WO/2005/ 003842 (2005).

10. J. Jarosz et al., "Adaptive eyeglasses for presbyopia correction: an original variable-focus technology," Opt. Express 27, 10533-10552 (2019).

11. G. Li et al., "Switchable electro-optic diffractive lens with high efficiency for ophthalmic applications," Proc. Natl. Acad. Sci. USA 103, 6100-6104 (2006).

12. Y. Yadin, A. Alon, and Y. Haddad, "Lenses with electrically-tunable power and alignment," WIPO patent WO/2014/049577 (2014).

13. M. Valley, "Flat liquid crystal diffractive lenses with variable focus and magnification," Doctor's Dissertation, The University of Arizona (2010).

14. V. Portney, "Refractive-diffractive switchable optical element," US Patent No: 9,364,319 (2016).

15. J. Schwiegerling, Field Guide to Visual and Ophthalmic Optics, SPIE Press, Bellingham, WA (2004). 
16. M. Jalie, "Modern spectacle lens design," Clin. Exp. Optom. 103(1), 3-10 (2020).

17. M. Jalie, "Best form lenses. Off-axis performance of lenses," Optom. Today 25, 24-31 (2005).

18. A. Mata, A. J. Fleischman, and S. Roy, "Characterization of polydimethylsiloxane (PDMS) properties for biomedical micro/nanosystems," Biomed. Microdevices 7, 281-293 (2005).

19. “TPX mechanical and optical characteristics," https://www.mitsui.com/de/en/business/ plastics/_icsFiles/afieldfile/2015/12/25/100408_TPX.pdf (accessed 3 November 2020).

20. J. N. Lee, C. Park, and G. M. Whitesides, "Solvent compatibility of poly(dimethylsiloxane)based microfluidic devices," Anal. Chern. 75, 6544-6554 (2003).

21. "Perfluorodecalin characteristics," http://www.fluoromed.com/products/perfluorodecalin/ (accessed 3 November 2020).

22. S. G. Kramer et al., "Perfluorocarbon liquids in ophthalmology," Surv. Ophthalmol. 39, 375-395 (1995).

23. R. L. McGee et al., "Densities, viscosities, refractive indexes, and molar refractions of the binary system tetraethylene glycol dimethyl ether-water at 25 degree C," J. Chem. Eng. Data 28, 305-307 (1983).

24. "Tetraethylene glycol characteristics," https://pubchem.ncbi.nlm.nih.gov/compound/ Tetraethylene-glycol (accessed 3 November 2020).

25. "Tetraethylene glycol characteristics," https://www.sigmaaldrich.com/catalog/product/ aldrich/110175?lang=en\&region=US (accessed 3 November 2020).

26. T. K. Kim, J. K. Kim, and O. C. Jeong, "Measurement of nonlinear mechanical properties of PDMS elastomer," Microelectron. Eng. 88, 1982-1985 (2011).

27. V. Portney, "New bi-sign aspheric IOL and its application," Optom. Vis. Sci. 89(1), 80-89 (2012).

28. "Multi-order diffractive optic," https://www.apollooptical.com/multi-order-diffractiveoptics/ (accessed 3 November 2020).

29. D. A. Buralli and G. M. Morris, "Effects of diffraction efficiency on the modulation transfer function of diffractive lenses," Appl. Opt. 31, 4389-4396 (1992).

30. M. E. Vlachopoulou et al., "A low temperature surface modification assisted method for bonding plastic substrates," J. Micromech. Microeng. 19, 15007-15016 (2009).

31. Y. H. Tennico et al., "Surface modification-assisted bonding of polymer-based microfluidic devices," Sens. Actuators B 143, 799-804 (2010).

32. H. Y. Tan, W. K. Loke, and N. T. Nguyen, "A reliable method for bonding polydimethylsiloxane (PDMS) to polymethylmethacrylate (PMMA) and its application in micropumps," Sens. Actuators B 151(1), 133-139 (2010).

33. M. M. Faghih and M. K. Sharp, "Solvent-based bonding of PMMA-PMMA for microfluidic applications," Microsyst. Technol. 25, 3547-3558 (2019).

34. R. Qin and C. Duan, "The principle and applications of Bernoulli equation," J. Phys. Conf. Ser. 916, 012038 (2017).

35. J. Armengol et al., "Bernoulli correction to viscous losses: radial flow between two parallel discs," Am. J. Phys. 76, 730-737 (2008).

36. M. S. Millán, F. Vega, and I. Ríos-López, "Polychromatic image performance of diffractive bifocal intraocular lenses: longitudinal chromatic aberration and energy efficiency," Invest. Ophthalmol. Vis. Sci. 57, 2021-2028 (2016).

37. "Noves eyewear," https://www.eschenbach.com/products/diagnostic-dispensing-systemsnoves-tray.asp (accessed 30 November 2020).

38. "Piezo engineering tutorial," https://www.aerotech.com/piezo-engineering-tutorial/ (accessed 18 April 2021).

39. M. Cognolato, M. Atzori, and H. Muller, "Head-mounted eye gaze tracking devices: an overview of modern devices and recent advances," J. Rehab. Assistive Technol. Eng. 5, 1-13 (2018).

40. "Optical lab knowledge for the non-lab optician," https://www.opticiantraining.com/wpcontent/uploads/2016/07/Surfacing-new-2.pdf (accessed 30 November 2020).

Valdemar Portney received his master's degree in physics from Moscow State University, specializing in optics and spectroscopy, and his $\mathrm{PhD}$ from Belarus State University in theoretical 
physics (quantum electrodynamics). In the U.S., his research interest has been in ophthalmic devices with an emphasis on multifocal optics, physiological optics, and instrumentation for optical testing. He is the holder of many patents and publications in corresponding areas. $\mathrm{He}$ is a member of the American Academy of Optometry.

F. Richard Christ received his bachelor's degree in chemistry and his master's degree in chemical engineering from the University of California, Los Angeles. His area of academic research related to porous media flow of aqueous polymeric solutions. Professionally, he has an extensive history in the development and evaluation of ophthalmic biomaterials and is the holder of more than 30 patents in that field. Currently, he is the president/chief executive officer of InVision Biomedical Group.

Marie Dvorak Christ received her bachelor's degree in chemical engineering from the University of California, Berkeley, and her PhD in chemistry from the University of California, Irvine. Her graduate research involved the elucidation of biopolymer molecular structure using nmr, mass spectrometry, and molecular modeling strategies. She is the chief scientific officer at InVision Biomedical Group, specializing in biomedical device materials development and biocompatibility testing. She holds ten patents in the field of biomaterials. 